\title{
Quasi-Periodicities Detection Using Phase-Rectified Signal Averaging in EEG Signals as a Depth of Anesthesia Monitor
}

\author{
Quan Liu, Yi-Feng Chen, Shou-Zen Fan, Maysam F. Abbod, and Jiann-Shing Shieh*
}

\begin{abstract}
Phase-rectified signal averaging (PRSA) has been known to be a useful method to detect periodicities in non-stationary biological signals. Determination of quasi-periodicities in electroencephalogram (EEG) is a candidate for quantifying the changes of depth of anesthesia (DOA). In this paper, DOA monitoring capacity of periodicities detected using PRSA were quantified by assessing EEG signals collected from 56 patients during surgery. The method is compared to sample entropy (SampEn), detrended fluctuation analysis (DFA) and permutation entropy (PE). The performance of quasi-periodicities defined by acceleration capacity (AC) and deceleration capacity (DC) was tested using the area under the receiver operating characteristic curve (AUC) and Pearson correlation coefficient. During the surgery, a significant difference $(p<0.05)$ in the quasi-periodicities was observed among three different stages under general anesthesia. There is a larger mean AUC and correlation coefficient of quasi-periodicities compared to SampEn, DFA and PE using expert assessment of conscious level (EACL) and bispectral index (BIS) as the gold standard, respectively. Quasi-periodicities detected using PRSA in EEG signals are powerful monitor of DOA and perform more accurate and robust results compared to SampEn, DFA and PE. The results do provide a valuable reference to researchers in the filed of clinical applications.
\end{abstract}

Index Terms - depth of anesthesia, electroencephalogram (EEG), phase-rectified signal averaging, quasi-periodicities.

Manuscript submitted December 26, 2015. This research is financially supported by the Center for Dynamical Biomarkers and Translational Medicine, National Central University, Taiwan which is sponsored by Ministry of Science and Technology (Grant Number: MOST103-2911-I-008-001). Also the work is supported by National Natural Science Foundation of China (Grants Numbers: 51475342 and 61201246). Asterisk indicates corresponding author.

Quan Liu is with the Key Laboratory of Fiber Optic Sensing Technology and Information Processing, Ministry of Education, Wuhan University of Technology, Wuhan, Hubei 430070 China (e-mail: quanliu@ whut.edu.cn).

Yi-Feng Chen is with the School of Information Engineering, Wuhan University of Technology, Wuhan, Hubei 430070 China (e-mail: yifengchen@whut.edu.cn).

Shou-Zen Fan is with the Department of Anesthesiology, College of Medicine, National Taiwan University, Taipei 100 Taiwan (e-mail: shouzen@gmail.com).

Maysam F. Abbod is with the Department of Electronic and Computer Engineering, Brunel University London, Uxbridge, UB8 3PH UK (e-mail: maysam.abbod@brunel.ac.uk).

*Jiann-Shing Shieh is with the Department of Mechanical Engineering, and Innovation Center for Big Data and Digital Convergence, Yuan Ze University, Taoyuan, Chung-Li 32003 Taiwan. (e-mail: jsshieh@saturn.yzu.edu.tw).

\section{INTRODUCTION}

$\bigcup_{s}$ ENERAL anesthesia is an indispensable procedure during Surgery to guarantee unconsciousness and total lack of sensation. In preparing for an operation, anesthesiologists are responsible for choosing and determining the optimal combination of anesthetic agents and dosages according to type of surgical procedure and the particular patient. To ensure patients' safety and a successful procedure, anesthetists need to adjust the dosage of anesthetics and sedatives based on patients' hypnotic state to avoid overdosing, and underdosing. Short et al. [1] have designed a balanced anesthesia study to determine whether causality exists between relatively deep anesthesia and the increased postoperative mortality. Several evidences indicate that excessive anesthetic due to overdosing can contribute to a potential hazardous despite a controversy over association with postoperative mortality [2-5], and inadequate anesthesia because of underdosing may cause intraoperative awareness and further lead to a potential incidence of postoperative psychosomatic dysfunction [6, 7]. Therefore, a reliable monitor to trace the change of anesthetic drug effects and determine depth of anesthesia (DOA) can help anesthesiologists to maintain adequate anesthesia with minimal agents.

Unfortunately, the biochemical mechanism of action of general anesthesia are still poorly understood [8, 9], and scientific definition of consciousness have not been accepted, thus is difficult to measure. Therefore, a golden standard directly associated with consciousness level for measurement of DOA remains not yet available [10]. To induce unconsciousness, anesthetic drugs act on myriad sites in the central nervous system (CNS) include the brain and spinal cord. The brain is the major functional unit of the central nervous system [11] which produce electroencephalogram (EEG) which reflects electrical activity of the brain. EEG have been widely used to determine the DOA $[10,12,13]$.

Currently, commercially EEG-based monitors mainly contain bispectral index (BIS) [14], Entropy index [15], patient state index [16] and Narcotrend index [17]. These monitors provide a single dimension index ranged from 0 (EEG silence) to 100 (awake) to indicate the DOA. The BIS, most popular used in the hospital, is a complex parameter derived from analysis in time domain, frequency domain and high order statistics [14]. Entropy monitoring, developed by Datex-Ohmeda, evaluates the complexity in EEG signals based on spectral entropy and provide two parameters (RE-Response 
Entropy, SE-State Entropy) associated with different frequency components [15]. In the patient state index, EEG power, frequency and phase information reflecting different dimensions of brain electrical activity are weighted combined to obtain a complex indicator [16]. Narcotrend uses a multivariate analysis algorithm to extract numerous quantitative features such as spectral parameters, entropy measures and autoregressive parameters from the time and the frequency domain in EEG [17]. However, the reliability of these monitors have been questioned in some special cases. BIS is insensitive to some commonly used drugs in general anesthesia such as ketamine and nitrous oxide [18, 19] and sensitive to artifacts [20]. In addition, its technology leads to a large time delay in the detection of the different stages of anesthesia [21]. The spectral entropy in entropy monitoring is calculated mainly in the frequency domain based on the power spectrum of the EEG using Fast Fourier Transformation (FFT). FFT as a linear algorithm is usually suitable for linear and stationary signals, but it cannot clearly reflect dynamic behavior in nonlinear and non-stationary EEG. Furthermore, Narcotrend does not have the ability to differentiate reliably between awareness and unconsciousness in the challenging data set from loss and return of consciousness [22], hence Narcotrend monitor ability to differentiate consciousness from unconsciousness for some agents is seriously doubted [23].

Recently, due to the nonlinear and non-stationary characteristics of EEG signals, sophisticated nonlinear dynamical approaches have been proposed to measure the DOA. Complexity measurement in EEG such as Lempel-Ziv complexity (LZC), approximate entropy (ApEn), sample entropy (SampEn), and permutation entropy (PE) provided a novel trend in the development of DOA monitoring [24, 25]. Detrended fluctuation analysis (DFA) has also been introduced to assess the scaling behavior in EEG as a measure of DOA [26, 27]. Nguyen-Ky et al. proposed a new method called wavelet-based depth of anesthesia (WDOA) index based on discrete wavelet transform (DWT) in 2011 [13] and then applied statistical method based on Bayesian techniques to analyze EEG signals for consciousness and DOA assessment in 2012 [28]. Hayashi et al. used SD1/SD2 ratio based on Poincaré plot to study dynamical changes of EEG induced by anesthesia in the state-space trajectory [29]. Furthermore, recurrence quantification analysis [30] and dimensionality reduction method [31] were also used to assess the depth of anesthesia.

In this paper, a technique called phase-rectified signal averaging (PRSA) is introduced to detect the quasi-periodicities in EEG for the assessment of DOA. PRSA was originally proposed by Bauer et al. to study quasi-periodic oscillations in noisy, non-stationary signals [32] and have been widely applied to analyzing heart rate variability (HRV) [33-35]. This is the first time PRSA is used to study the quasi-periodicities in EEG as a measure of DOA. In human being, CNS continuously receives internal regulations and noxious stimuli under the control of brain and processing this information to determine the correct response and then sending the information to body. During general anesthesia, the loops are interrupted or changed causing anesthesia induced unconsciousness [36, 37]. Quasiperiodic oscillations in EEG reflect regulation processes of the CNS. The EEG originating from multiple regions of the brain such as cerebral cortex and thalamus is of high complexity and always contaminated by artifacts. While PRSA has advantages in the analysis of nonlinear and non-stationary signals over conventional spectral methods and can eliminating artifacts or noise. Furthermore, PRSA provides a way to analyze periodic behavior that is related to acceleration and deceleration in EEG during anesthesia, which offers a possibility for anesthesia monitoring. In the present study, the acceleration capacity (AC) and deceleration capacity (DC) of EEG signals are quantified for monitoring the DOA. The results are compared to the gold standard indictor decided by five experienced anesthesiologists known as expert assessment of conscious level (EACL) [38, 39] and bispectral index (BIS) [39].

\section{MATERIALS AND METHODS}

\section{A. Data Sources}

A single channel raw EEG data and BIS were collected from 56 ASA physical status I-III patients (age 18-75 years) during surgery in operation room using the MP60 system (Philip, IntelliVue MP60 BIS module). The patients are undergoing ear, nose, and throat (ENT) surgery at the National Taiwan University Hospital (NTUH) of Taiwan. Normally, EEG recording begins before surgery starts and ends after patient's awareness recovery. Before starting the drug administration, all patients were awake and had awareness. Then they all received propofol and fentanyl for induction of anesthesia. Muscle relaxants were also administered at the discretion of the anesthesiologist. Sevoflurane, desflurane and propofol were administered individually by an anesthesiologist for maintenance of anesthesia. The corresponding anesthetic technique are sevoflurane for endotracheal intubation for 11 patients, desflurane for endotracheal intubation for 13 patients, sevoflurane for laryngeal mask airway (LMA) for 16 patients, desflurane for laryngeal mask airway (LMA) of 4 patients, and propofol for total intravenous anesthesia of 12 patients, respectively. At the end of surgery, sevoflurane, desflurane and propofol were discontinued. Then the patients were asked to be wakened from anesthesia state. The sampling rate of EEG is $125 \mathrm{~Hz}$ and the BIS is $0.2 \mathrm{~Hz}$. The present research received the approval of Institutional Review Board at NTUH of Taiwan. Written informed consents were obtained from the 56 patients.

\section{B. Expert Assessment of Conscious Level}

Expert assessments of conscious level (EACL) are derived from five experienced anesthesiologists from NTUH in Taiwan to trace patients' state of anesthetic depth [38, 39]. During the whole duration of the surgery, two nurses continuously observed the patients' state and recorded the vital signs possibly associated with drug effects on patients. The operation type, clinical indices include blood pressure, heart rate and $\mathrm{SpO} 2$, the detailed information of operation events happened during surgery such as induction, extubation and drugs administration, the type of anesthetics and their dose, and MAC values were all recorded on the operation record during the whole period of anesthesia. The age, weight, height, gender and medical history of patients were also recorded. The anesthesiologists took the record into consideration and made their own decision independently based on their previous experiences to plot the state of anesthetic depth of patients 
$>$ This article has been accepted for publication in a future issue of this journal, but has not been fully edited. Content may change prior to final

between 0 (i.e., EEG silence) and 100 (i.e., awake) consisting with commonly used monitors. Other continuous signs were digitized at a sample rate of $0.2 \mathrm{~Hz}$ such as BIS. Each person of the five anesthesiologists can provide different value for the state of anesthesia depth at the same time, therefore, the mean value was obtained from the five EACL decided by anesthesiologists for increasing reliability and accuracy. Because these selected five doctors have worked as anesthesiologists for many years, the EACL are reliable and can be used to as a real gold standard for the recognition of different anesthesia states of patients. Fig. 1 shows an example of EACL from five doctors and their mean and standard deviation.

\section{Phase-Rectified Signal Averaging}

Phase-rectified signal averaging (PRSA) is a recently introduced signal processing technique to analyze non-stationary and nonlinear time series contaminated by noise or artifacts [32]. It has been widely used for analysis of acceleration capacity (AC) and deceleration capacity (DC)

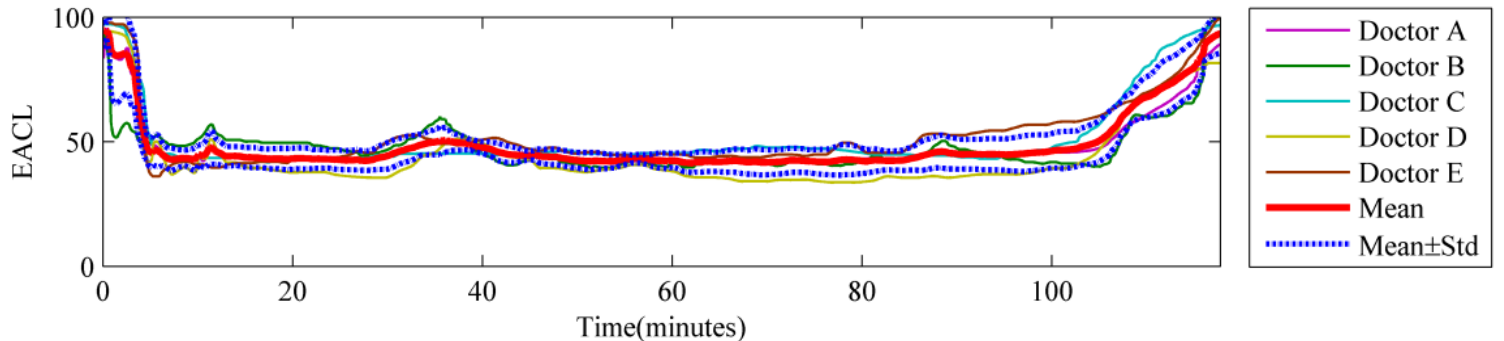

Fig. 1. EACL of one patient from five anesthesiologists and the corresponding mean and standard deviation of five EACL.

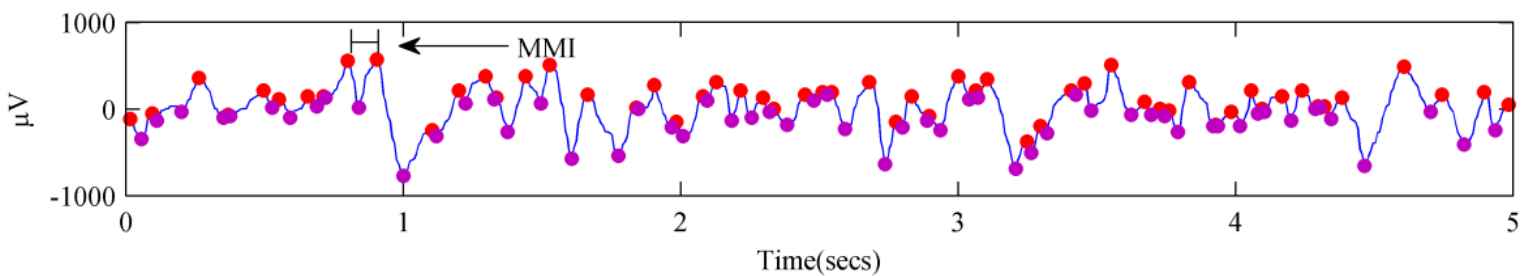

(a)

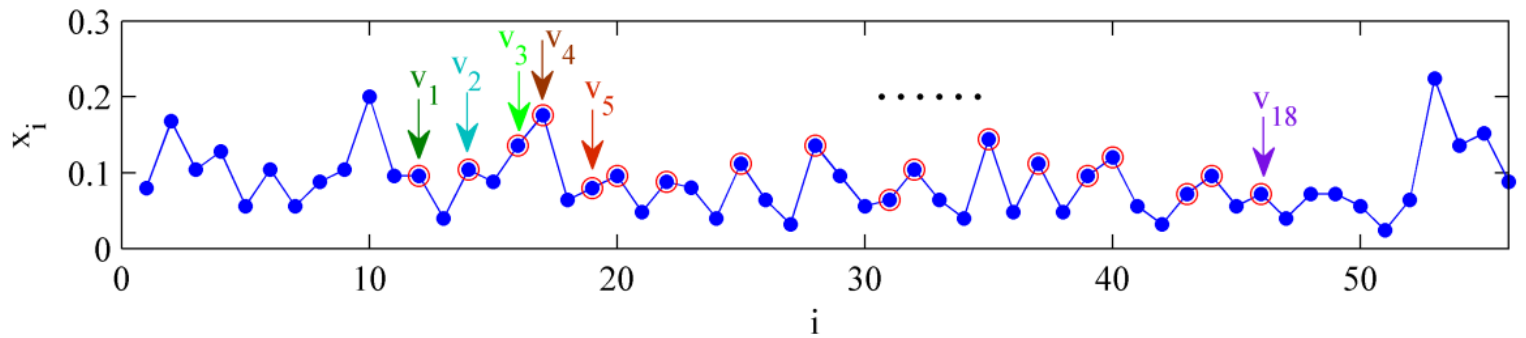

(b)

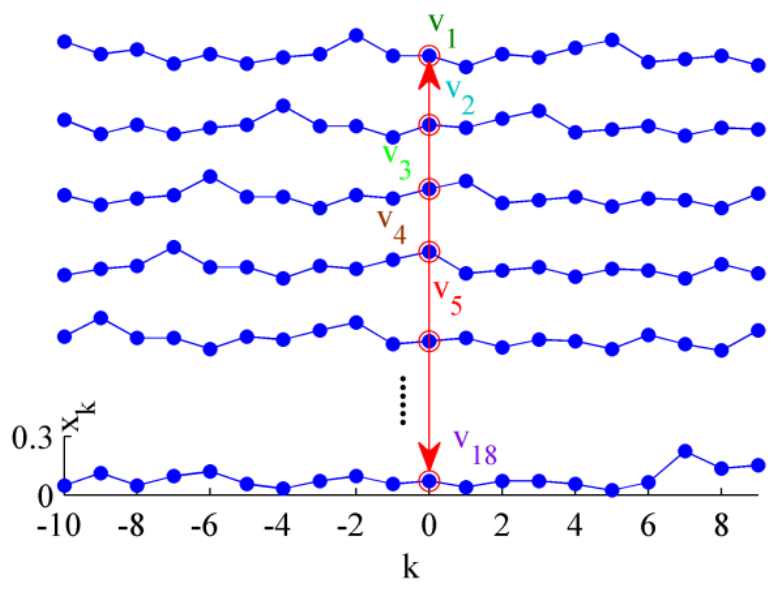

(c)

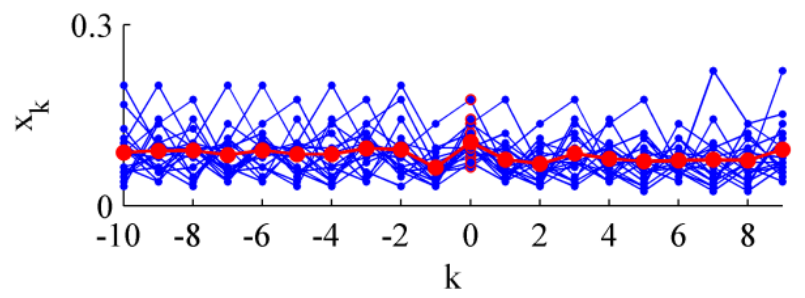

(d)

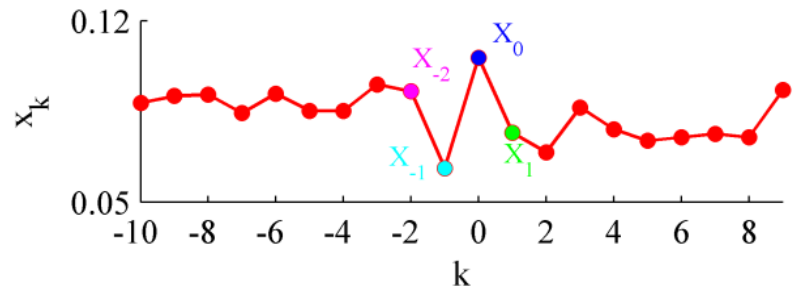

(e)

Fig. 2. Illustrations of PRSA. (a) Definition of MMI in EEG. (b) Anchor points detection and definition related to increase events. (c) Surroundings alignment around each anchor point. (d) PRSA curve by averaging over all aligned surroundings. (e) Quantification of AC or DC.

of heart rate [33]. In this paper, PRSA is used on EEG signals to detect the quasi-periodicities by deceleration capacity and acceleration capacity. Fig. 2 provides a detailed interpretation of PRSA for studying EEG. Firstly the maxima and minima in EEG segments are detected and transferred the EEG data to calculate the maximum-to-maximum or minimum-to-minimum 
$>$ This article has been accepted for publication in a future issue of this journal, but has not been fully edited. Content may change prior to final publication. Citation information: DOI10.1109/TNSRE.2017.2690449, IEEE Transactions on Neural Systems and Rehabilitation Engineering

interval (MMI) which is the time interval between successive maxima or minima. Fig. 2(a) shows the maxima and minima detection and MMI definition. Fig. 2(b) shows the MMI sequences extracted from EEG. For AC calculation, the increased points in MMI sequences are selected as anchors (i.e., $\left.v_{1}, v_{2}, v_{3}, v_{4}, v_{5}, \ldots, v_{18}\right)$ as shown in Fig. 2(b). The decreased points in MMI sequences are selected as anchors for DC calculation. Surroundings of window length $2 \mathrm{~L}$ around the anchor points are selected and L is set to be 10 in Fig. 2(c), then the extracted surroundings are aligned at the anchor points. The PRSA curve is derived from averaging over all aligned surroundings as shown in Fig. 2(d) marked with red line. Finally, the AD and DC are quantified as follows:

$$
A C(D C)=\frac{X_{0}+X_{1}-X_{-1}-X_{-2}}{4}
$$

where $X_{0}$ is the average of MMI at all anchor points, $X_{1}$ is the following point adjacent to $X_{0}$ in PRSA curve, $X_{-1}$ and $X_{-2}$ are preceding points adjacent to $X_{0}$ as shown in Fig. 2(e).

Mathematically, the quantification of AC and DC is based wavelet transform associated with the Haar wavelet defined as (2) [32]. The discrete wavelet transform applied to PRSA signal at time scale $\mathrm{s}$ and position $p$ is generally expressed with (3) [32]. It captures both frequency and location information, and the wavelet coefficients along time scale $s$ indicate the characteristics of periodicities or frequencies in the signals. Actually, the AC and DC are central wavelet amplitude $x_{\phi}(2,0)$ calculated using Haar wavelet analysis where $s=2$ and $p=0$. Hence, they carry the time scales information of the periodicities in the PRSA signal. In the procedure of PRSA, non-periodic components are cancel out due to the averaging of all aligned surroundings around the anchor points, thus this calculation is robust to noise or artifacts and can be applied to extract information about the quasi-periodicities from complex signals which maybe non-stationary and noisy.

$$
\begin{gathered}
\phi(t)=\left\{\begin{array}{cc}
1 & 0 \leq t<1 \\
-1 & -1 \leq t<0 \\
0 & \text { otherwise }
\end{array}\right. \\
x_{\phi}(s, p)=\sum_{k=-L}^{L-1} x_{k} \phi[(k-p) / s]
\end{gathered}
$$

\section{EMD/MEMD based Filter}

Generally, EEG records electrical signals from activity of the brain along the scalp and almost always contaminated by biological and environmental artifacts, such as EOG, EMG, power line interference and baseline drift and so on. And the amplitude of artifacts can be highly larger than the signals of interest. Therefore, it is necessary to remove them from EEG before analysis for the purpose of accuracy. Empirical mode decomposition (EMD) introduced by Huang et al. [40] has been widely used in analysis of non-stationary and nonlinear signals. EMD decomposes the original signals into finite components called intrinsic mode functions (IMFs) from high to low frequency adaptively by a sifting process given by:

$$
x(t)=\sum_{i=1}^{N} I M F_{i}(t)+r_{N}(t)
$$

where $x(t)$ is the original signal, $N$ is the total number of IMFs, $I M F_{i}(t)$ is the $i$ th component, and $r_{N}(t)$ is the residual. Then, the filtered signal $\hat{x}(t)$ can be reconstructed using selected IMFs dominated by signal of interest [41, 42], as follows:

$$
\begin{gathered}
\hat{x}(t)=\sum_{i=p}^{q} I M F_{i}(t) \quad(1 \leq p \leq q \leq N) \text { or } \\
\hat{x}(t)=\sum_{i=1}^{q} I M F_{i}(t)+\sum_{i=p}^{N} I M F_{i}(t) \quad(1 \leq q \leq p \leq N)
\end{gathered}
$$

Multivariate empirical mode decomposition (MEMD) as an improved algorithm of EMD was proposed to decompose multichannel signals [43, 44]. In order to solve the mode mixing problem, MEMD was applied in this study to decompose a three channel signal consisting of one channel EEG and two channels independent white noise. Then the IMFs corresponding to EEG channel are extracted. According to previous researches, the authors $[39,45,46]$ set $\mathrm{p}=2, \mathrm{q}=3$ to reconstruct the filtered EEG signals. Therefore, $I M F_{2}(t)$ and $I M F_{3}(t)$ were selected as the filtered EEG in this paper.

\section{E. Statistical Analyses}

To assess the performance of proposed quasi-periodicities quantification by AC and DC in EEG for DOA measurement, statistical analyses were used. Firstly, two-tailed Student's t-tests were performed to indicate whether there is a significant difference to distinguish different anesthesia states. Then AC and DC were compared with EACL and BIS, respectively. The Pearson correlation coefficient was applied to assess the correlation between AC (DC) and EACL and BIS, respectively. This was used to evaluate the ability of AC and DC to track the changes of patients' state undergoing anesthesia. In addition, receiver operating characteristic (ROC) analysis was used and area under the receiver operating characteristic curve (AUC) of $\mathrm{AC}$ and DC was calculated to illustrate their performance to monitor DOA. The two-tailed Student's t-tests and ROC analysis were performed in SPSS (v19, SPSS Inc.; Chicago, IL, USA). The level of statistical significance was set at $\mathrm{p}<0.05$.

The whole diagram for monitoring the DOA based on the proposed method is shown in Fig. 3. The raw EEG signal is firstly filtered using MEMD method. Then the signal is reconstructed by summing up IMF2 and IMF3 as the filtered signal [39]. After that the $\mathrm{AC}$ and $\mathrm{DC}$ indices are extracted based on PRSA method from the signal to assess the DOA. The values of AC and DC have different scales from EACL and BIS, therefore, both of them are converted to the same scale as EACL and BIS via an artificial neural network (ANN). The output of ANN is used to monitor the DOA. The ANN topology is 1-20-1 neurons for the input layer, hidden layer and output layer respectively. The network is feed-forward back-propagation type. Each of the parameters including AC and DC was used as input and EACL or BIS was used as target data, respectively to train, validate and test the ANN model. The samples percentages divided randomly for training, validation, and testing are $70 \%, 15 \%$, and $15 \%$, respectively. The training data set is firstly used to train the neural network. During training, current values of the weights and biases are applied to the validation data set and the error on the validation set is monitored to avoid model overfitting. The test data is not used at all during the training process. After training completes, the accuracy of the resulting neural network model's weights 
$>$ This article has been accepted for publication in a future issue of this journal, but has not been fully edited. Content may change prior to final publication. Citation information: DOI10.1109/TNSRE.2017.2690449, IEEE Transactions on Neural Systems and Rehabilitation Engineering $>$ and biases are applied to the test data. The ANN network was implemented in MATLAB (v7.13, MathWorks, Inc., USA).

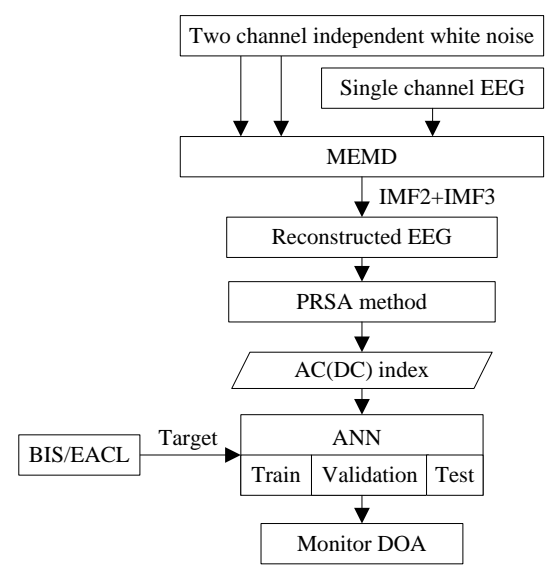

Fig. 3. Flow chart for monitoring the DOA based on PRSA method

\section{RESULTS}

\section{A. Capacity of AC and DC for Distinguishing Different Stages during Surgery}

In general, the general anesthesia can be divided into pre-operation (i.e., stage 1), induction and maintenance (i.e., stage 2) and recovery (i.e., stage 3 ) during the whole surgical procedure. Patients are awake and keep a high consciousness level during the pre-operation. Then induction of general

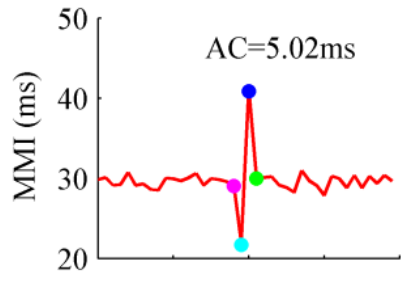

(a)

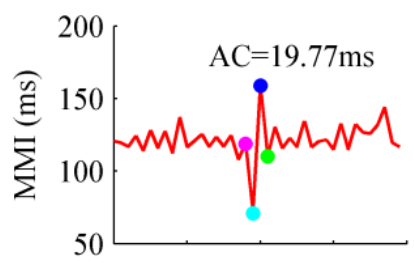

(e)

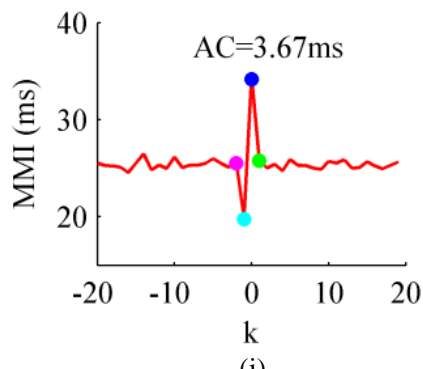

(i)

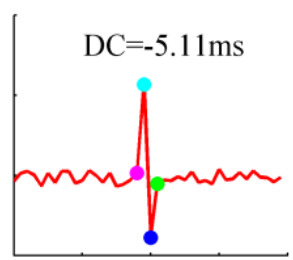

(b)

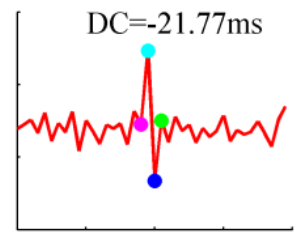

(f)

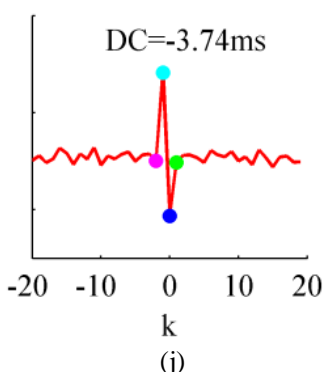

anesthesia is conducted and they totally lose their consciousness. Anesthesia must be continuously maintained in order to avoid recovery of consciousness until the operation is finished. After the operation, the concentration of administered agent drops, patients recover their awareness and consciousness.

First, the behavior of AC and DC to distinguish the states under different anesthesia stages is investigated. Fig. 4 presents the acceleration and deceleration-related PRSA signals of MMI of EEG recording in a patient under stage 1, stage 2 and stage 3 from top to bottom, respectively. All oscillations are in phase at the anchor point, thus AC and DC are the sum of the all periodic oscillations corresponding to acceleration and deceleration. In this patient, AC and DC provide a clear index to indicate the DOA under three different stages, and the absolute values of both $\mathrm{AC}$ and DC are largest in the middle line (i.e., stage 2) when the patient is under anesthesia without awareness and consciousness. Both AC and DC calculated from maximum-to-maximum or minimum-to-minimum interval of EEG recording are substantially symmetric and correspond in absolute value.

The statistical distribution of AC and DC for each stage from 56 patients undergoing general anesthesia is presented in Fig. 5. It can be seen that the absolute values of both AC and DC clearly increase from stage 1 to stage 2 , and then decrease from stage 2 to stage 3 . They increase when patients lose their awareness and consciousness and decrease with recovery.

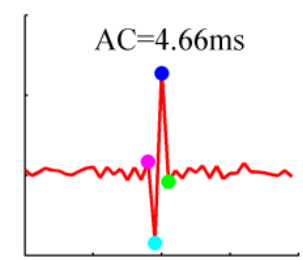

(c)

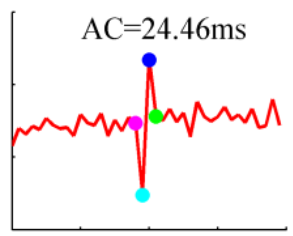

(g)

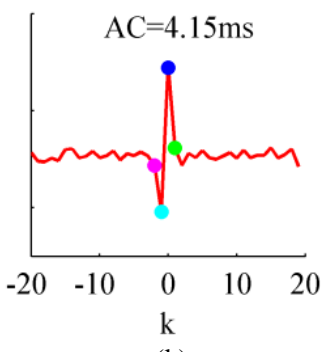

(k)

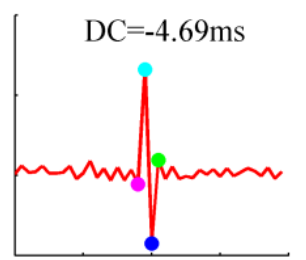

(d)

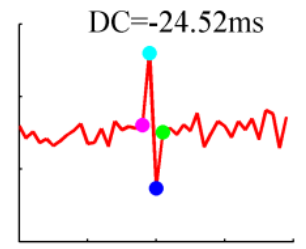

(h)

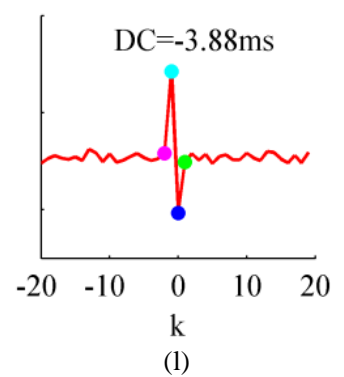

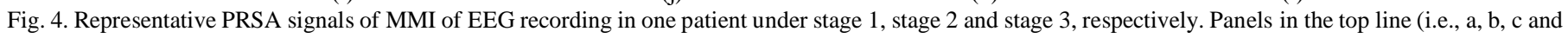

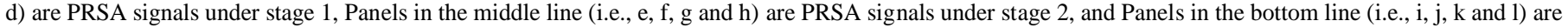

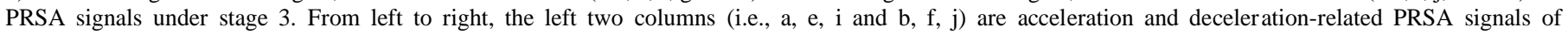

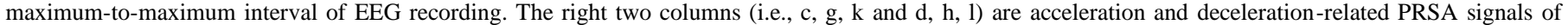
minimum-to-minimum interval of EEG recordings. 

publication. Citation information: DOI10.1109/TNSRE.2017.2690449, IEEE Transactions on Neural Systems and Rehabilitation Engineering

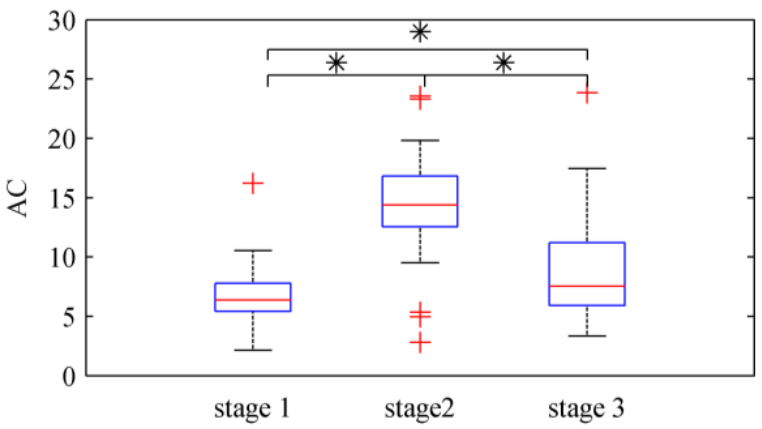

(a)

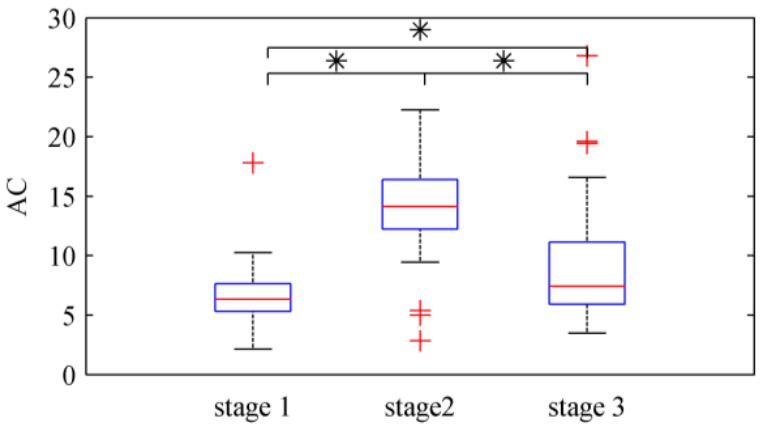

(c)

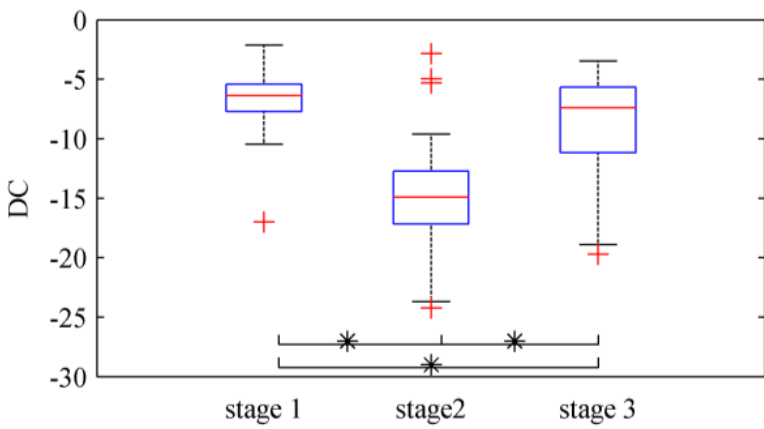

(b)

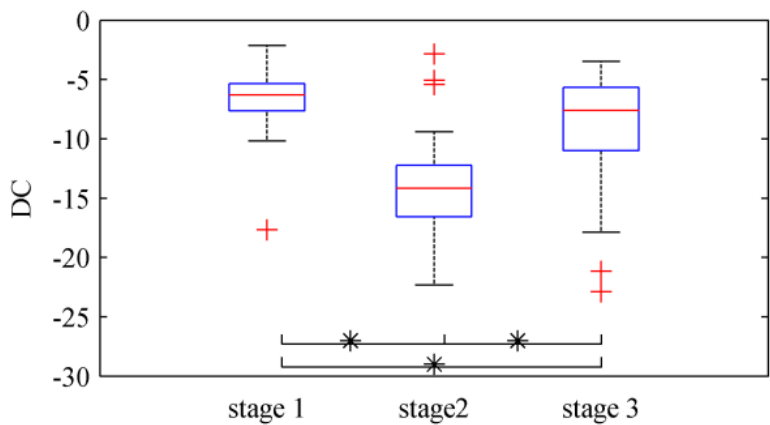

(d)

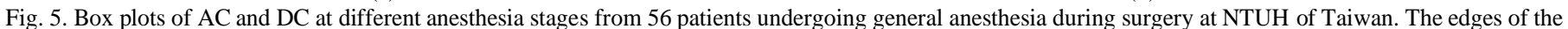

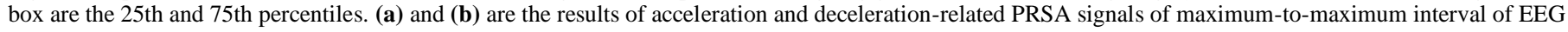

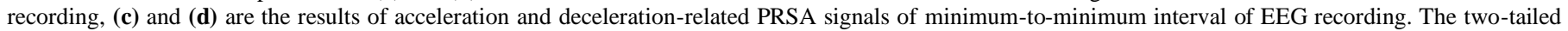
Student's t-tests are used to indicate the significant difference between two different stages. Those giving p-value lower that 0.05 are marked with asterisk (*).

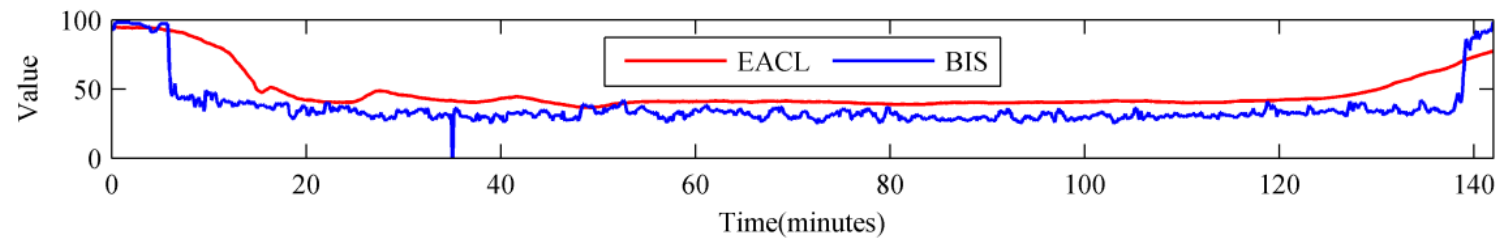

(a)

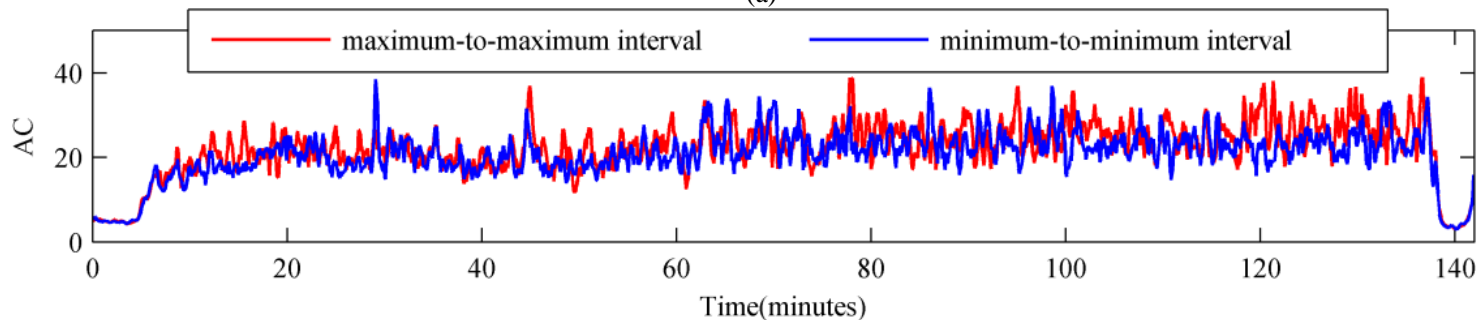

(b)

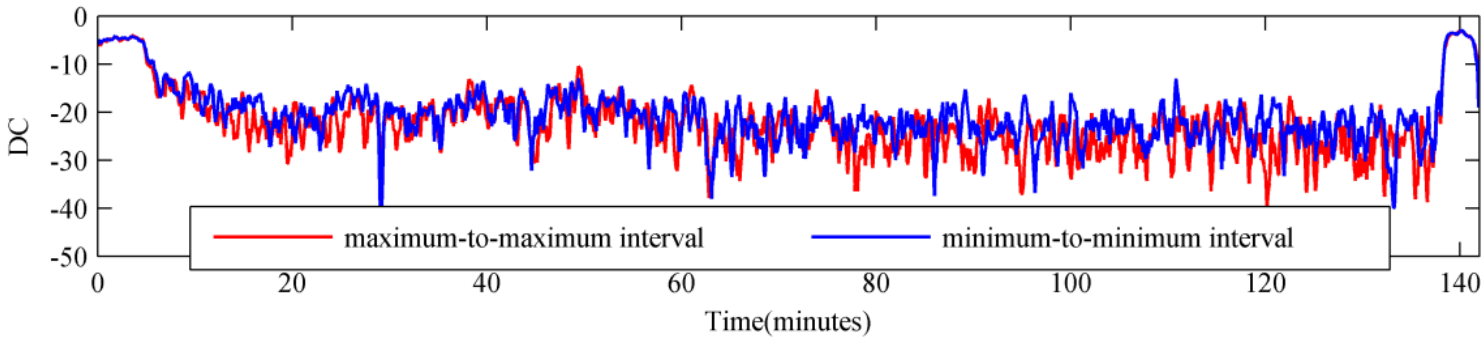

(c)

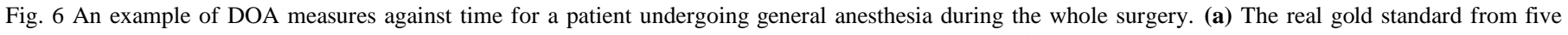

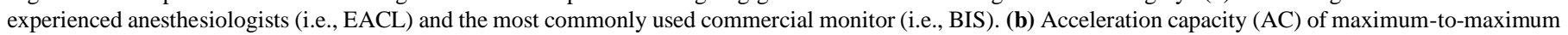
and minimum-to-minimum interval. (c) Deceleration capacity (DC) of maximum-to-maximum and minimum-to-minimum interval. 
$>$

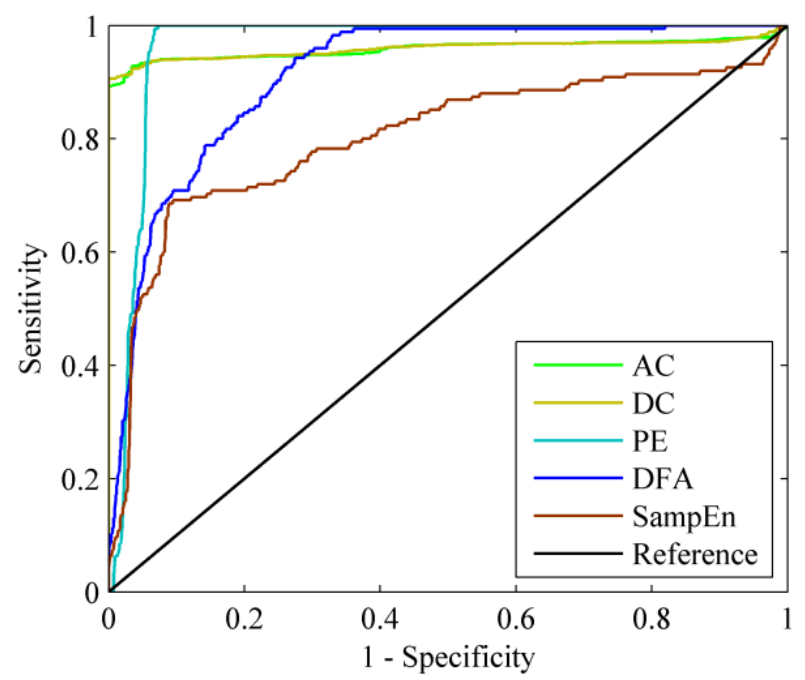

Fig. 7 ROC curves for prediction DOA by AC, DC, SampEn, DFA and PE using EACL as gold standard. $\mathrm{AC}=$ acceleration capacity of maximum-to-maximum interval, $\mathrm{DC}=$ deceleration capacity of maximum-to-maximum interval, SampEn=sample entropy, DFA=detrended fluctuation analysis and $\mathrm{PE}=$ permutation entropy.

Furthermore, $\mathrm{p}<0.05$ indicates that there is a statistically significant difference of both AC and DC between any two different stages; therefore, both AC and DC can distinguish stage 1, stage 2 and stage 3 from each other for measuring the consciousness level correctly.

\section{B. Capacity of $A C$ and $D C$ for Tracing the Consciousness Level during Surgery}

In this section, PRSA is used to trace the consciousness level of 56 patients undergoing general anesthesia during surgery. The AC and DC indicators are compared with a real gold standard from five experienced anesthesiologists (i.e., EACL) and the most commonly used commercial monitor (i.e., BIS) for monitoring changes in anesthesia states of patients. Because EACL and BIS are sampled at $0.2 \mathrm{~Hz}$ and the sampling rate of EEG data is $125 \mathrm{~Hz}$, therefore a fixed-size sliding window moving once every $5 \mathrm{~s}$ with 625 points was used, and the window size was set to be 3750 data points with duration of 30 s according to our previous studies [39, 46]. An example of anesthesia state monitoring of one patient undergoing general anesthesia during surgery is shown in Fig. 6. AC values which are inverse to EACL and BIS trend increase with losing awareness and consciousness under anesthesia of the patient, and then decrease during recovery when the anesthesia states is getting lighter. Larger AC values indicate deeper anesthesia level. On the contrary, the DC values consisting with EACL and BIS trend changes from high values to low values when patient's states change from awake to anesthesia.

The performance of the proposed method was compared with other existing algorithms, such as sample entropy (SampEn), detrended fluctuation analysis (DFA) [27] and permutation entropy (PE) $[25,47]$. The detailed algorithms and parameters selection of SampEn and PE were illustrated in our previous study [46]. In calculation of SampEn, the value of embedding dimension $m$ is set to 2 and the value of tolerance $r$ is set to $0.2 \times s t d$, where $s t d$ stands for standard deviation. The DFA is calculated according to the illustration in [27].

To evaluate the performance of the proposed method for tracing the DOA, ROC analysis is carried out. AUC is a commonly used measure in medical diagnosis referred to as the discrimination. EACL and BIS were used to determine the anesthesia and awake states. In general, the BIS value ranged from 40 to 60 indicates an appropriate anesthesia level during surgery, thus the threshold to identify anesthesia state is set to be 60. The value of EACL and BIS below 60 is defined as anesthesia state and value above 60 is defined as awake state. Fig. 7 shows a graphical plot of ROC that illustrates the performance of AC, DC, SampEn, DFA and PE for anesthesia discrimination. The best classification has the largest AUC. The AUC of AC and DC is larger than that of SampEn, DFA and PE. The SampEn yields the lowest AUC. This result suggests that classification based on both AC and DC has an improved predictive power in discriminating awake state from anaesthesia state in comparison with SampEn, DFA and PE. The mean and standard deviation values of AUC of AC, DC, SampEn, DFA and PE from 56 patients are shown in TABLE I. The AC and DC have a high discrimination of the difference between anesthesia state and awake state. The mean values of AUC of AC and DC are significantly larger than that of SampEn, DFA and PE ( $p<0.05)$ before and after regression via ANN.

Furthermore, Pearson correlation coefficient between AC, DC, SampEn, DFA and PE and EACL, BIS was tested as shown in TABLE II. The correlation coefficients of AC, DC, SampEn, DFA and PE increase after regression via ANN. The mean values of correlation coefficients of AC and DC are larger than that of SampEn, DFA and PE. In particular, AC and DC have a better linear correlation with EACL in comparison with BIS, therefore they are more consistent with the real gold standard for DOA monitoring. Based on these observations mentioned above, the capacity of AC and DC is significantly better than that of SampEn, DFA and PE for tracing the change of DOA.

TABLE I

STATISTICAL RESUlts of AUC OF ROCS FOR AC, DC, SAMPEN, DFA AND PE FrOM 56 PATIENTS. THE VALUES ARE PRESENTED WITH MEAN (SD). THE ORIGINAL EEG SIGNALS WERE USED WITHOUT FILTERING. BOTH AC AND DC OF MAXIMUM-TO-MAXIMUM AND MINIMUM-TO-MINIMUM INTERVAL (MMI) EXTRACTED FROM EEG WERE CALCULATED TO TEST THEIR PERFORMANCE USING EACL AND BIS AS GOLD STANDARD. THOSE GIVING P-VALUE LOWER THAT 0.05 OF DIFFERENCE FROM SAMPEN, DFA AND PE ARE MARKED WITH ASTERISK $(*)$, DAGGER $(\dagger)$ AND DOUBLE DAGGER $(\dot{\dagger})$, RESPECTIVELY.

\begin{tabular}{|c|c|c|c|c|c|c|c|}
\hline \multicolumn{8}{|c|}{ Without ANN } \\
\hline & MAXI_AC & MAXI_DC & MINI_AC & MINI_DC & SampEn & DFA & PE \\
\hline vs. EACL & $0.90(0.07) *+$ & $0.90(0.08) * \dagger$ & $0.90(0.08) * \dagger+$ & $0.89(0.08) * t+$ & $0.49(0.19)$ & $0.70(0.21)$ & $0.81(0.16)$ \\
\hline vs. BIS & $0.90(0.08) * t$ & $0.91(0.07) * \dagger$ & $0.91(0.07) *+$ & $0.91(0.07) *+$ & $0.57(0.19)$ & $0.68(0.20)$ & $0.85(0.14)$ \\
\hline
\end{tabular}




\begin{tabular}{|c|c|c|c|c|c|c|c|}
\hline vs. EACL & $0.94(0.07) * \dagger$ & $0.94(0.07) * \dagger$ & $0.91(0.09) * \dagger$ & $0.91(0.09) * \dagger$ & $0.75(0.15)$ & $0.79(0.13)$ & $0.84(0.10)$ \\
\hline vs. BIS & $0.93(0.06) * \dagger$ & $0.94(0.06) *$ & $0.92(0.07) *+\$$ & $0.93(0.07) * \dagger$ & $0.71(0.14)$ & $0.75(0.16)$ & $0.88(0.09)$ \\
\hline
\end{tabular}

TABLE II

Statistical Results of Pearson correlation coefficient Between AC, DC, SAmpEn, DFA, PE and EACL, BIS From 56 PATIENTS. The VAlues are PRESENTED WITH MEAN (SD). THE ORIGINAL EEG SIGNALS WITHOUT FILTERING WERE USED. THOSE GIVING P-VALUE LOWER THAT 0.05 OF DIFFERENCE FROM SAMPEN, DFA AND PE ARE MARKED WITH ASTERISK $(*)$, DAGGER $(\dagger)$ AND DOUBLE DAGGER $(\$)$, RESPECTIVELY.

\begin{tabular}{|c|c|c|c|c|c|c|c|}
\hline \multicolumn{8}{|c|}{ Without ANN } \\
\hline & MAXI_AC & MAXI_DC & MINI_AC & MINI_DC & SampEn & DFA & PE \\
\hline vs. EACL & $0.59(0.13) * \dagger$ & $0.59(0.14) * \dagger$ & $0.59(0.14) * \dagger$ & $0.58(0.14) * \dagger$ & $0.05(0.31)$ & $0.25(0.31)$ & $0.47(0.28)$ \\
\hline vs. BIS & $0.51(0.27) * \dagger$ & $0.51(0.28) *^{\dagger \dagger}$ & $0.51(0.27) * \dagger$ & $0.51(0.27) * \dagger$ & $0.18(0.26)$ & $0.15(0.30)$ & $0.48(0.28)$ \\
\hline \multicolumn{8}{|c|}{ With ANN } \\
\hline vs. EACL & $0.77(0.12) *{ }^{2}$ & $0.77(0.13) *+$ & $0.76(0.13) * \dagger$ & $0.76(0.13) *+4$ & $0.44(0.23)$ & $0.52(0.27)$ & $0.65(0.20)$ \\
\hline vs. BIS & $0.59(0.33) * \dagger$ & $0.59(0.33) *^{\dagger}$ & $0.58(0.32) * \dagger$ & $0.58(0.33) * \dagger$ & $0.37(0.19)$ & $0.43(0.26)$ & $0.58(0.18)$ \\
\hline
\end{tabular}

\section{Capacity of $A C$ and $D C$ for Artifacts Rejection during Surgery}

It is noted that the amplitude of EEG signal is very weak and almost always contaminated by noise or artifacts. Therefore, a good enough EEG-based indicator should be insensitive to noise or artifacts for accuracy in measurement of DOA. In this section, AC, DC, SampEn, DFA and PE after filtering using MEMD method were compared with that before filtering mentioned in the previous section to evaluate their sensitivity to artifacts in EEG.

TABLE III presents the statistical result of AUC of ROCs for AC, DC, SampEn, DFA and PE from 56 patients' EEG signals after filtering. Those giving $\mathrm{p}$-value indicate the statistically significant difference level compared with the corresponding index before filtering. The $\mathrm{AC}$ and $\mathrm{DC}$ still present a high discrimination of the difference between anesthesia state and awake state and yield a larger AUC than SampEn, DFA and PE after filtering. Furthermore, it can be seen in $4^{\text {th }}$ row in
TABLE III that the p-values of AC and DC (i.e., 0.61, 0.71, 0.84 and 0.53 ) using EACL as the gold standard without ANN have no statistically significant difference $(p>0.05)$ compared with TABLE I before filtering. The larger p-values in the $6^{\text {th }}$ row in
TABLE III indicate that the significance of the difference for $\mathrm{AC}$ and DC (i.e., 0.04, 0.04, 0.04, 0.08) between before and after filtering using BIS as the gold standard without ANN is less than that for SampEn (i.e., 0.00). There is a statistically significant improvement $(p<0.05)$ for SampEn in discrimination of the difference between anesthesia state and awake state. The AC and DC of minimum-to-minimum interval perform no significant difference between before and after filtering using EACL and BIS as the gold standard after regression via $\mathrm{ANN}$. $\mathrm{AC}$ and $\mathrm{DC}$ of maximum-to-maximum after regression via ANN preserve a higher discrimination after filtering than SampEn, DFA and PE in spite of a difference compared with before filtering. Therefore, the measure of $\mathrm{AC}$ and DC in EEG are less sensitive to noise or artifacts and better than SampEn, DFA and PE for DOA monitoring. Especially, the p-values of AC and DC using EACL as gold standard in $4^{\text {th }}$ and $9^{\text {th }}$ rows in 
TABLE III are larger than that using BIS as gold standard in $6^{\text {th }}$ and $11^{\text {th }}$ rows in
TABLE III which indicates that the proposed method is more robust to determine the anesthesia state using the real gold standard (i.e., EACL).

In addition, TABLE IV presents the Pearson correlation coefficient between AC, DC, SampEn, DFA and PE and EACL, BIS after filtering, respectively. The correlation coefficients of AC and DC are clearly significantly larger $(\mathrm{p}<$ $0.05)$ than SampEn and DFA. There is a high linear correlation (i.e., $0.78 \pm 0.12$ ) between AC, DC and EACL. The SampEn are more close to the EACL and BIS trend after filtering. The p-values in $9^{\text {th }}$ row (i.e., 0.09, 0.11, 0.08 and 0.08) in TABLE IV show that the AC and DC have no significant difference between before and after filtering using EACL as the gold standard after regression via ANN. The AC and DC are more close to EACL with higher correlation coefficients than BIS trend consisting with the conclusion as mentioned above.

TABLE III

THE STATISTICAL RESUlt OF AUC OF ROCS FOR AC, DC, SAMPEN, DFA AND PE FROM 56 PATIENTS. THE VALUES ARE PRESENTED WITH MEAN (SD). THE EEG SIGNALS AFTER FILTERING WERE USED. THOSE GIVING P-VALUE LOWER THAT 0.05 OF DIFFERENCE FROM SAMPEN, DFA AND PE ARE MARKED WITH ASTERISK (*), DAGGER $(\dagger)$ AND DOUBLE DAGGER $(\dagger)$, RESPECTIVELY. THE P-VALUE IN THE TABLE INDICATES THE STATISTICAL SIGNIFICANT DIFFERENCE LEVEL COMPARED WITH BEFORE FILTERING.

\begin{tabular}{|c|c|c|c|c|c|c|c|}
\hline \multicolumn{8}{|c|}{ Without ANN } \\
\hline & MAXI_AC & MAXI_DC & MINI_AC & MINI_DC & SampEn & DFA & $\mathrm{PE}$ \\
\hline vs. EACL & $0.90(0.09) * \dagger$ & $0.90(0.09) * \dagger$ & $0.90(0.09) * \dagger$ & $0.90(0.09) * \dagger$ & $0.73(0.14)$ & $0.66(0.12)$ & $0.88(0.11)$ \\
\hline $\mathrm{p}$-value & 0.61 & 0.71 & 0.84 & 0.53 & 0.00 & 0.16 & 0.00 \\
\hline vs. BIS & $0.88(0.10) * \dagger$ & $0.89(0.10) * \dagger$ & $0.88(0.10) * \dagger$ & $0.89(0.10) * \dagger$ & $0.79(0.15)$ & $0.66(0.10)$ & $0.87(0.12)$ \\
\hline $\mathrm{p}$-value & 0.04 & 0.04 & 0.04 & 0.08 & 0.00 & 0.61 & 0.09 \\
\hline \multicolumn{8}{|c|}{ With ANN } \\
\hline vs. EACL & $0.92(0.08) * \dagger$ & $0.92(0.08) * \dagger$ & $0.91(0.08) * \dagger$ & $0.92(0.07) *^{\dagger}$ & $0.83(0.11)$ & $0.78(0.11)$ & $0.86(0.13)$ \\
\hline $\mathrm{p}$-value & 0.04 & 0.03 & 0.66 & 0.46 & 0.00 & 0.12 & 0.09 \\
\hline vs. BIS & $0.91(0.09) * \dagger$ & $0.91(0.09) * \dagger$ & $0.90(0.09) * \dagger$ & $0.92(0.08) * \dagger$ & $0.80(0.15)$ & $0.79(0.12)$ & $0.89(0.10)$ \\
\hline p-value & 0.00 & 0.01 & 0.05 & 0.28 & 0.00 & 0.08 & 0.26 \\
\hline
\end{tabular}

TABLE IV

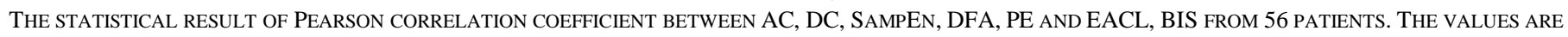
PRESENTED WITH MEAN (SD). THE EEG SIGNALS AFTER FILTERING WERE USED. THOSE GIVING P-VALUE LOWER THAT 0.05 OF DIFFERENCE FROM SAMPEN, DFA AND PE ARE MARKED WITH ASTERISK $(*)$, DAGGER $(\dagger)$ AND DOUBLE DAGGER $(\dagger)$, RESPECTIVELY. THE P-VALUE IN THE TABLE INDICATES THE STATISTICAL SIGNIFICANT DIFFERENCE LEVEL COMPARED WITH THE CORRESPONDING INDEX BEFORE FILTERING.

\begin{tabular}{cccccccc}
\hline \hline \multicolumn{7}{c}{ Without ANN } \\
\hline & MAXI_AC & MAXI_DC & MINI_AC & MINI_DC & SampEn & DFA & PE \\
vs. EACL & $0.64(0.14) * *^{\dagger}$ & $0.64(0.14) * \dagger$ & $0.64(0.14) * \dagger$ & $0.64(0.14) * \dagger$ & $0.25(0.29)$ & $0.18(0.19)$ & $0.61(0.21)$ \\
p-value & 0.00 & 0.00 & 0.00 & 0.00 & 0.00 & 0.05 & 0.00 \\
\hline \hline
\end{tabular}


> This article has been accepted for publication in a future issue of this journal, but has not been fully edited. Content may change prior to final publication. Citation information: DOI10.1109/TNSRE.2017.2690449, IEEE Transactions on Neural Systems and Rehabilitation Engineering

\begin{tabular}{cccccccc}
\hline \hline vs. BIS & $0.48(0.32) * \dagger$ & $0.47(0.32) * \dagger$ & $0.47(0.32) * \dagger$ & $0.47(0.32) * \dagger$ & $0.40(0.23)$ & $0.17(0.17)$ & $0.48(0.33)$ \\
p-value & 0.03 & 0.01 & 0.01 & 0.01 & 0.00 & 0.71 & 0.84 \\
& \multicolumn{7}{c}{ With ANN } \\
vs. EACL & $0.78(0.12)^{* \dagger+}$ & $0.78(0.12) * *+$ & $0.78(0.12) * \dagger+$ & $0.78(0.12) * *+$ & $0.55(0.18)$ & $0.20(0.14)$ & $0.69(0.19)$ \\
p-value & 0.09 & 0.11 & 0.08 & 0.08 & 0.00 & 0.15 & 0.09 \\
vs. BIS & $0.54(0.36)^{* \dagger}$ & $0.54(0.37) * \dagger$ & $0.53(0.36) * \dagger$ & $0.54(0.37) * \dagger$ & $0.50(0.24)$ & $0.16(0.14)$ & $0.58(0.21)$ \\
p-value & 0.00 & 0.00 & 0.01 & 0.02 & 0.00 & 0.12 & 0.15 \\
\hline \hline
\end{tabular}

\section{DISCUSSIONS}

General anesthesia acts on CNS and blocks the functional connection between thalamic and cortical and subcortical areas which has been suggested to be the reason of unconsciousness induced by anesthesia [36, 37, 48]. Thus, the loops are interrupted of human body for extracting, receiving, processing and sending the information from internal or external stimuli under anesthesia. There will be a depression of signaling in the brain produced by agents [49], so the quasi-periodicities of EEG signal will increase. On the other hand, EEG activity oscillates at a various range of frequencies; these oscillations tend to be higher in amplitude and slower in frequency under general anesthesia. Therefore, when a patient is under anesthesia state, there is a tendency for quasi-periodicities in EEG signal to become larger. It is confirmed by our findings that the absolute values of $\mathrm{AC}$ and $\mathrm{DC}$ of EEG recording increase when patients lose their awareness and consciousness produced by anesthesia agents. The AC and DC yield a significant difference to distinguish stage 1, stage 2 and stage 3 during the whole general anesthesia. Furthermore, the larger AUC and correlation coefficient values indicate that the quasi-periodicities defined by $\mathrm{AC}$ and $\mathrm{DC}$ provide a strong indicator of DOA during surgery and is better than PE, DFA and SampEn in the case of accuracy.

The PRSA technique synchronizes the phases of all periodic components of the signal irrespective of their frequencies or characteristic time scales. It thus integrates their contributions by accumulation of the corresponding amplitudes at the centre of the PRSA signal. Quasi-periodic and periodic sinusoidal modes in a contaminated signal will result in anchor points predominantly in the phase of the steepest increase or decrease, i.e. the phase is 0 or $\pi$ [32]. In this case, it is a data driven method to obtain the phase information of the oscillations and anchor points can phase-rectify the signal. In the average, non-periodic components that are not phase synchronized with the anchor points, i.e., non-stationarities, artifacts, and noise cancel out, and events that have a fixed phase relationship with the anchor points, i.e., all periodicities and quasi-periodicities, are reserved by the procedure. Therefore, the PRSA technique has advantage in noisy, non-stationary signals, artifacts could be eliminated by averaging segments aligned at the anchors. $\mathrm{AC}$ and DC perform less sensitive to artifacts in EEG signal.

In this paper, EACL and BIS were used to define the awake and anesthesia states and target data for ANN model, respectively. As we known, the BIS index is highly sensitive to artifacts, it may fail to detect consciousness in poor signal quality [28]. In this case, the reliability of using BIS as the gold standard is inadequate than that of using EACL, which is proved by our findings in TABLE IV that there are larger correlation coefficient values using EACL as the gold standard. So our proposed method can be used as a more accurate reference of the DOA estimation based on EACL.

There are various limitations in this study. First, burst suppression EEG pattern occurring during deep anesthesia was not taken into consideration. Burst suppression is a serious problem in the DOA monitors based on EEG, which can be regarded as light anesthesia level in spite of under deep anesthesia [50]. BIS index has solved this problem by introducing a ratio to indicate burst suppression behavior [51]. In current study, no burst suppression was found in the analyzed EEG collected from 56 patients. So there is no impact on our findings. However, future works still are needed to confirm whether the proposed method can detect burst suppression pattern and is robust to measure the DOA accurately. Second, three type of agents including propofol, sevoflurane and desflurane were used to induce and maintain an appropriate anesthesia level in 56 patients, and they have the same mechanisms to causes anesthesia by gamma-aminobutyric acid $\left(\mathrm{GABA}_{\mathrm{A}}\right)$ receptor to depress CNS activity $[49,52]$. So our results support the agents with the same the mechanism of action. Nevertheless, several anesthetic agents such as ketamine and nitrous oxide have different mechanism to produce general anesthesia, so whether the proposed method can be applied to measure anesthesia with the agent-specific mechanism also needs more potential works to confirm.

\section{CONCLUSION}

This paper study a PRSA technique to detect the quasi-periodicities of EEG signal defined by $\mathrm{AC}$ and $\mathrm{DC}$ parameters for DOA monitoring under general anesthesia. First, maximum-to-maximum and minimum-to-minimum intervals (MMI) of the original EEG signal are extracted. So the abnormal absolute amplitude of EEG due to artifacts have less impact on the accuracy. Second, PRSA was used to process extracted MMI sequences from EEG, the phases of all periodic segments are synchronized by alignment at the anchor points, and the artifacts can be eliminated by segments averaging. There is a significant difference (i.e., $\mathrm{p}<0.05$ ) for AC and DC to distinguish stage 1 , stage 2 and stage 3 during the whole general anesthesia. The proposed method provides a strong discrimination between anesthesia and awake states and good correlation with EACL via ANN. Furthermore, we also compared their performance before and after filtering using the sum of $I M F_{2}(t)$ and $I M F_{3}(t), \mathrm{AC}$ and DC perform better than SampEn, DFA and PE in the case of accuracy and robustness to measure the DOA under general anesthesia. 
It is noted that there is a stronger correlation between $\mathrm{AC}, \mathrm{DC}$ and EACL than BIS in spite of similar AUC. The EACL as the real gold standard derived from five experienced anesthesiologists provides a reliable target to define and trace the consciousness level. It would not have the drawbacks occurring in BIS. Consequently, the $\mathrm{AC}$ and $\mathrm{DC}$ after regression via ANN using EACL as target are more acceptable and present a real DOA monitor ranged from 0 to 100 to indicate the anesthesia trend.

\section{REFERENCES}

T. G. Short, K. Leslie, M. T. Chan, D. Campbell, C. Frampton, and P. Myles, "Rationale and design of the balanced anesthesia study: a prospective randomized clinical trial of two levels of anesthetic depth on patient outcome after major surgery," Anesthesia \& Analgesia, vol. 121, pp. 357-365, 2015.

[2] M. D. Kertai, P. Nirvik, B. J. A. Palanca, L. Nan, S. A. Searleman, Z. Lini, et al., "Association of Perioperative Risk Factors and Cumulative Duration of Low Bispectral Index with Intermediate-term Mortality after Cardiac Surgery in the B-Unaware Trial," Anesthesiology, vol. 112, pp. 1116-1127, 2010.

[3] M. D. Kertai, B. J. A. Palanca, P. Nirvik, B. A. Burnside, Z. Lini, S. Furqaan, et al., "Bispectral index monitoring, duration of bispectral index below 45, patient risk factors, and intermediate-term mortality after noncardiac surgery in the B-Unaware Trial," Anesthesiology, vol. 114, pp. 545-556, 2011.

[4] M. Lindholm, S. Traff, F, S. Greenwald, A. Ekbom, C. Lennmarken, and R. Sandin, "Mortality within 2 years after surgery in relation to low intraoperative bispectral index values and preexisting malignant disease," Anesthesia \& Analgesia, vol. 108, pp. 508-512, 2009.

[5] T. G. Monk, B. C. Saini VWeldon, and J. C. Sigl, "Anesthetic management and one-year mortality after noncardiac surgery," Anesthesia \& Analgesia, vol. 100, pp. 4-10, 2005.

[6] K. Leslie and M. P. Chan, "Posttraumatic stress disorder in aware patients from the B-aware trial," Anesthesia \& Analgesia, vol. 110, pp. 823-828, 2010.

[7] P. S. Sebel, B. T Andrew, M. M. Ghoneim, I. J. Rampil, R. E. Padilla, G. T. Joo, et al., "The incidence of awareness during anesthesia: a multicenter United States study," Anesthesia \& Analgesia, vol. 99, pp. 833-839, 2004.

[8] H. C. H. Jr, M. H. Akabas, P. A. Goldstein, J. R. Trudell, B. A. Orser, and N. L. Harrison, "Emerging molecular mechanisms of general anesthetic action," Trends in Pharmacological Sciences, vol. 26, pp. 503-510, 2005.

[9] C. P-L, "New insights into the molecular mechanisms of general anaesthetics," British Journal of Pharmacology, vol. 161, pp. 288-307, 2010.

[10] P. Gifani, H. R. Rabiee, M. H. Hashemi, P. Taslimi, and M. Ghanbari, "Optimal fractal-scaling analysis of human EEG dynamic for depth of anesthesia quantification," Journal of the Franklin Institute, vol. 344, pp. 212-229, 2007.

[11] J. Kiernan and R. Rajakumar, Barr's the human nervous system: an anatomical viewpoint: Lippincott Williams \& Wilkins, 2013.

[12] M. Kreuzer, E. F. Kochs, G. Schneider, and D. Jordan, "Non-stationarity of EEG during wakefulness and anaesthesia: advantages of EEG permutation entropy monitoring," Journal of Clinical Monitoring \& Computing, vol. 28, pp. 573-580, 2014.

[13] T. Nguyen-Ky, P. Wen, Y. Li, and R. Gray, "Measuring and reflecting depth of anesthesia using wavelet and power spectral density," Information Technology in Biomedicine, IEEE Transactions on, vol. 15, pp. 630-639, 2011.

[14] I. J. Rampil, "A primer for EEG signal processing in anesthesia," Anesthesiology, vol. 89, pp. 18-23, 1998.

[15] H. Viertiö-Oja, V. Maja, M. Särkelä, P. Talja, N. Tenkanen, H. Tolvanen-Laakso, et al., "Description of the Entropy ${ }^{\mathrm{TM}}$ algorithm as applied in the Datex-Ohmeda S/5 $5^{\mathrm{TM}}$ Entropy Module," Acta Anaesthesiologica Scandinavica, vol. 48, pp. 154-161, 2004.

[16]

[17]

[24]
D. Drover and H. R. Ortega, "Patient state index," Best Practice \& Research Clinical Anaesthesiology, vol. 20, pp. 121-128, 2006.
S. Kreuer and W. Wilhelm, "The narcotrend monitor," Best Practice \& Research Clinical Anaesthesiology, vol. 20, pp. 111-119, 2006. G. Barr, J. G. Jakobsson, A. Owall, and R. E. Anderson, "Nitrous oxide does not alter bispectral index: study with nitrous oxide as sole agent and as an adjunct to i.v. anaesthesia," British Journal of Anaesthesia, vol. 82, pp. 827-830, 1999.

J. W. Johansen and P. S. Sebel, "Development and clinical application of electroencephalographic bispectrum monitoring," Anesthesiology, vol. 93, pp. 1336-1344, 2000.

M. Messner, U. Beese, J. Romstöck, M. Dinkel, and K. Tschaikowsky, "The bispectral index declines during neuromuscular block in fully awake persons," Anesthesia \&

Analgesia, vol. 97, pp. 488-491, 2003.
S. Pilge, R. Zanner, G. Schneider, J. Blum, M. Kreuzer, and E. F. Kochs, "Time delay of index calculation: analysis of cerebral state, bispectral, and narcotrend indices," Anesthesiology, vol. 104, pp.

488-494, 2006.
G. Schneider, E. F. Kochs, B. Horn, M. Kreuzer, and M. Ningler, "Narcotrend $®$ does not adequately detect the transition between awareness and unconsciousness in surgical patients," The Journal of the American Society of Anesthesiologists, vol. 101, pp. 1105-1111,

2004.
I. Russell, "The Narcotrend 'depth of anaesthesia'monitor cannot reliably detect consciousness during general anaesthesia: an investigation using the isolated forearm technique," British journal

of anaesthesia, vol. 96, pp. 346-352, 2006.
R. Ferenets, T. Lipping, A. Anier, V. Jäntti, S. Melto, and S. Hovilehto, "Comparison of entropy and complexity measures for the assessment of depth of sedation," Biomedical Engineering,

IEEE Transactions on, vol. 53, pp. 1067-1077, 2006.
$\mathrm{X}$. Li, S. Cui, and L. J. Voss, "Using permutation entropy to measure the electroencephalographic effects of sevoflurane," Anesthesiology, vol. 109, pp. 448-456, 2008.

M. Jospin, P. Caminal, E. W. Jensen, H. Litvan, M. Vallverdú, M. M. Struys, et al., "Detrended fluctuation analysis of EEG as a measure of depth of anesthesia," Biomedical Engineering, IEEE Transactions on, vol. 54, pp. 840-846, 2007.

T. Nguyen-Ky, P. Wen, and Y. Li, "Improving the accuracy of depth of anaesthesia using modified detrended fluctuation analysis method," Biomedical Signal Processing and Control, vol. 5, pp. 59-65, 2010.

T. Nguyen-Ky, P. Wen, and Y. Li, "Consciousness and depth of anesthesia assessment based on bayesian analysis of EEG signals," Biomedical Engineering, IEEE Transactions on, vol. 60, pp. 1488-1498, 2013.

K. Hayashi, N. Mukai, and T. Sawa, "Poincaré analysis of the electroencephalogram during sevoflurane anesthesia," Clinical Neurophysiology, vol. 126, pp. 404-411, 2015.

R. Shalbaf, H. Behnam, J. W. Sleigh, D. A. Steyn-Ross, and M. L. Steyn-Ross, "Frontal-temporal synchronization of eeg signals quantified by order patterns cross recurrence analysis during propofol anesthesia," IEEE Transactions on Neural Systems and Rehabilitation Engineering, vol. 23, pp. 468-474, 2015.

M. Mirsadeghi, H. Behnam, R. Shalbaf, and H. J. Moghadam, "Characterizing awake and anesthetized states using a dimensionality reduction method," Journal of medical systems, vol. 40, pp. 1-8, 2016.

A. Bauer, J. W. Kantelhardt, A. Bunde, P. Barthel, R. Schneider, M. Malik, et al., "Phase-rectified signal averaging detects quasi-periodicities in non-stationary data," Physica A: Statistical Mechanics and its Applications, vol. 364, pp. 423-434, 2006.

A. Bauer, J. W. Kantelhardt, P. Barthel, R. Schneider, T. Mäkikallio, K. Ulm, et al., "Deceleration capacity of heart rate as a predictor of mortality after myocardial infarction: cohort study," The lancet, vol. 367, pp. 1674-1681, 2006.

L. M. Campana, R. L. Owens, G. D. Clifford, S. D. Pittman, and A. Malhotra, "Phase-rectified signal averaging as a sensitive index of autonomic changes with aging," Journal of Applied Physiology, vol. 108, pp. 1668-1673, 2010.

J. W. Kantelhardt, A. Bauer, A. Y. Schumann, P. Barthel, R. Schneider, M. Malik, et al., "Phase-rectified signal averaging for the detection of quasi-periodicities and the prediction of cardiovascular risk," Chaos: An Interdisciplinary Journal of Nonlinear Science, vol. 17, pp. 383-409, 2007. 
This article has been accepted for publication in a future issue of this journal, but has not been fully edited. Content may change prior to final publication. Citali2n information: DOI10.1109/TNSRE.2017.2690449, IEEE Transactions on Neural Systems and Rehabilitation Engineering

[36] M. Alkire, R. Haier, and J. Fallon, "Toward a unified theory of narcosis: brain imaging evidence for a thalamocortical switch as the neurophysiologic basis of anesthetic-induced unconsciousness," Consciousness and cognition, vol. 9, pp. 370-386, 2000.

[37] T. Gili, N. Saxena, A. Diukova, K. Murphy, J. E. Hall, and R. G. Wise, "The thalamus and brainstem act as key hubs in alterations of human brain network connectivity induced by mild propofol sedation," The Journal of Neuroscience, vol. 33, pp. 4024-4031, 2013.

[38] G. J. A. Jiang, S.-Z. Fan, M. F. Abbod, H.-H. Huang, J.-Y. Lan, F.-F. Tsai, et al., "Sample Entropy Analysis of EEG Signals via Artificial Neural Networks to Model Patients' Consciousness Level Based on Anesthesiologists Experience," BioMed Research International, vol. 2015, p. 8, 2015.

[39] Q. Liu, Y.-F. Chen, S.-Z. Fan, M. F. Abbod, and J.-S. Shieh, "EEG Signals Analysis Using Multiscale Entropy for Depth of Anesthesia Monitoring during Surgery through Artificial Neural Networks," Computational and Mathematical Methods in Medicine, vol. 2015, p. 16, 2015.

[40] N. E. Huang, Z. Shen, S. R. Long, M. C. Wu, H. H. Shih, Q. Zheng, et al., "The empirical mode decomposition and the Hilbert spectrum for nonlinear and non-stationary time series analysis," in Proceedings of the Royal Society of London A: Mathematical, Physical and Engineering Sciences, 1998, pp. 903-995.

[41] P. Flandrin, G. Rilling, and P. Goncalves, "Empirical mode decomposition as a filter bank," Signal Processing Letters, IEEE, vol. 11, pp. 112-114, 2004

[42] Z. Wu and N. E. Huang, "A study of the characteristics of white noise using the empirical mode decomposition method," in Proceedings of the Royal Society of London A: Mathematical, Physical and Engineering Sciences, 2004, pp. 1597-1611.

[43] N. Rehman and D. P. Mandic, "Multivariate empirical mode decomposition," in Proceedings of The Royal Society of London A: Mathematical, Physical and Engineering Sciences, 2009, pp. 1291-1302.
[44] N. U. Rehman and D. P. Mandic, "Filter bank property of multivariate empirical mode decomposition," Signal Processing, IEEE Transactions on, vol. 59, pp. 2421-2426, 2011.

[45] J.-R. Huang, S.-Z. Fan, M. F. Abbod, K.-K. Jen, J.-F. Wu, and J.-S. Shieh, "Application of multivariate empirical mode decomposition and sample entropy in EEG signals via artificial neural networks for interpreting depth of anesthesia," Entropy, vol. 15, pp. 3325-3339, 2013.

[46] Q. Liu, Y.-F. Chen, S.-Z. Fan, M. F. Abbod, and J.-S. Shieh, "A comparison of five different algorithms for EEG signal analysis in artifacts rejection for monitoring depth of anesthesia," Biomedical Signal Processing and Control, vol. 25, pp. 24-34, 2016.

[47] R. Shalbaf, H. Behnam, J. W. Sleigh, A. Steyn-Ross, and L. J. Voss, "Monitoring the depth of anesthesia using entropy features and an artificial neural network," Journal of neuroscience methods, vol. 218, pp. 17-24, 2013.

[48] N. P. Franks, "General anaesthesia: from molecular targets to neuronal pathways of sleep and arousal," Nature Reviews Neuroscience, vol. 9, pp. 370-386, 2008.

[49] M. C. Bieda, H. Su, and M. B. MacIver, "Anesthetics discriminate between tonic and phasic gamma-aminobutyric acid receptors on hippocampal CA1 neurons," Anesthesia and analgesia, vol. 108, pp. 484-490, 2009.

[50] J. Bruhn, H. Röpcke, B. Rehberg, T. Bouillon, and A. Hoeft, "Electroencephalogram approximate entropy correctly classifies the occurrence of burst suppression pattern as increasing anesthetic drug effect," Anesthesiology, vol. 93, pp. 981-985, 2000.

[51] J. C. Sigl and N. G. Chamoun, "An introduction to bispectral analysis for the electroencephalogram," Journal of clinical monitoring, vol. 10, pp. 392-404, 1994.

[52] D. Tanelian, "The role of the GABA_A receptor/chloride channel complex in anesthesia," Anesthesiology, vol. 78, pp. 757-776, 1993. 